\title{
Robust Nonlinear Model Predictive Control
}

\section{for Two-Stage Anaerobic Digesters}

\section{(Supporting Information)}

Ennio R. Piceno-Díaz ${ }^{1}$, Luis A. Ricardez-Sandoval², Miguel A. Gutierrez-Limon ${ }^{1 *}$, Hugo O. MéndezAcosta $^{3}$, and Héctor Puebla ${ }^{1}$

${ }^{1}$ Departamento de Energía, Universidad Autónoma Metropolitana-Azcapotzalco, Ciudad de

México, México

${ }^{2}$ Department of Chemical Engineering, University of Waterloo, Waterloo, Ontario, Canada

${ }^{3}$ Centro Universitario de Ciencias Exactas e Ingeniería, Universidad de Guadalajara, Jalisco, México

*magl@azc.uam.mx 


\begin{tabular}{|c|c|c|c|c|c|c|c|c|c|c|c|c|c|c|}
\hline$N s=5$ & $\begin{array}{l}\text { wi, } \\
\text { Case } \\
\text { (1) }\end{array}$ & $\begin{array}{l}\text { wi, } \\
\text { Case } \\
\text { (5) }\end{array}$ & $\mathrm{Ns}=10$ & $\begin{array}{l}\text { wi, } \\
\text { Case } \\
\text { (1) }\end{array}$ & $\begin{array}{l}\text { wi, } \\
\text { Case } \\
\text { (10) }\end{array}$ & $\mathrm{Ns}=15$ & $\begin{array}{l}\text { wi, } \\
\text { Case } \\
\text { (1) }\end{array}$ & $\begin{array}{l}\text { wi, } \\
\text { Case } \\
(15)\end{array}$ & $\alpha_{1}$ & $\alpha_{2}$ & $\mu_{11 \max }$ & $\mu_{12 \max }$ & $\mathrm{ks}_{12}$ & $\mu_{22 \max }$ \\
\hline \multirow[t]{3}{*}{1} & 0.5 & 0.05 & 1 & 0.35 & 0.01 & 1 & 0.005 & 0.45 & 0.085 & 0.25 & 0.15 & 0.35 & 5 & 0.22 \\
\hline & & & 2 & 0.25 & 0.01 & 2 & 0.005 & 0.2 & 0.093 & 0.27 & 0.17 & 0.4 & 4.75 & 0.23 \\
\hline & & & & & & 3 & 0.01 & 0.06 & 0.1 & 0.3 & 0.19 & 0.45 & 4.5 & 0.25 \\
\hline \multirow[t]{4}{*}{2} & 0.2 & 0.1 & 3 & 0.15 & 0.02 & 4 & 0.01 & 0.05 & 0.110 & 0.32 & 0.21 & 0.5 & 4.25 & 0.26 \\
\hline & & & 4 & 0.07 & 0.03 & 5 & 0.02 & 0.05 & 0.115 & 0.34 & 0.23 & 0.53 & 4.07 & 0.268 \\
\hline & & & & & & 6 & 0.02 & 0.04 & 0.12 & 0.35 & 0.25 & 0.55 & 3.88 & 0.275 \\
\hline & & & 5 & 0.06 & 0.05 & 7 & 0.02 & 0.03 & 0.125 & 0.365 & 0.27 & 0.57 & 3.69 & 0.28 \\
\hline \multirow[t]{4}{*}{3} & 0.15 & 0.15 & 6 & 0.05 & 0.06 & 8 & 0.03 & 0.03 & 0.13 & 0.38 & 0.29 & 0.6 & 3.5 & 0.29 \\
\hline & & & & & & 9 & 0.03 & 0.03 & 0.143 & 0.39 & 0.3 & 0.62 & 3.33 & 0.31 \\
\hline & & & 7 & 0.03 & 0.07 & 10 & 0.03 & 0.02 & 0.155 & 0.4 & 0.31 & 0.65 & 3.13 & 0.33 \\
\hline & & & & & & 11 & 0.07 & 0.01 & 0.167 & 0.41 & 0.33 & 0.68 & 2.94 & 0.35 \\
\hline \multirow[t]{3}{*}{4} & 0.1 & 0.2 & 8 & 0.02 & 0.15 & 12 & 0.07 & 0.01 & 0.18 & 0.42 & 0.34 & 0.7 & 2.75 & 0.37 \\
\hline & & & 9 & 0.01 & 0.25 & 13 & 0.08 & 0.01 & 0.187 & 0.44 & 0.36 & 0.73 & 2.5 & 0.4 \\
\hline & & & & & & 14 & 0.25 & 0.005 & 0.194 & 0.46 & 0.38 & 0.77 & 2.25 & 0.42 \\
\hline 5 & 0.05 & 0.5 & 10 & 0.01 & 0.35 & 15 & 0.35 & 0.005 & 0.2 & 0.47 & 0.41 & 0.8 & 2 & 0.44 \\
\hline Nominal & & & & & & & & & 0.13 & 0.38 & 0.27 & 0.5 & 3.5 & 0.29 \\
\hline \% Devmin & & & & & & & & & 35 & 24 & 44 & 30 & 43 & 24 \\
\hline \%Devmax & & & & & & & & & 54 & 34 & 52 & 60 & 43 & 52 \\
\hline
\end{tabular}

Table S1 Bounds of uncertain parameters and realizations or scenarios of the two-stage AD unit 


\begin{tabular}{|c|c|c|c|c|c|c|c|}
\hline \multirow[b]{2}{*}{ Param. } & \multirow[b]{2}{*}{$\begin{array}{l}\text { Plant } \\
\text { Cases }\end{array}$} & \multicolumn{2}{|c|}{ SSE } & \multicolumn{2}{|c|}{$\begin{array}{l}\text { Stable control output, } \mathrm{x} 6 \\
(\mathrm{mg} / \mathrm{dL})\end{array}$} & \multicolumn{2}{|c|}{$\begin{array}{c}\% \text { Output error with respect to set } \\
\text { point }\end{array}$} \\
\hline & & $\begin{array}{l}\text { Robust } \\
\text { NMPC }\end{array}$ & Std. NMPC & Robust NMPC & Std. NMPC & Robust NMPC & Std. NMPC \\
\hline \multirow{5}{*}{$\mu_{12 \max }$} & $(1)$ & 1.716 & 3.332 & 1.060 & 1.091 & $6.00 \%$ & $9.10 \%$ \\
\hline & (2) & 1.712 & 1.202 & 1.039 & 1.000 & $3.90 \%$ & $0.00 \%$ \\
\hline & (3) & 2.455 & 4.000 & 0.946 & 0.914 & $-5.38 \%$ & $-8.56 \%$ \\
\hline & (4) & 8.598 & 11.574 & 0.853 & 0.828 & $-14.70 \%$ & $-17.23 \%$ \\
\hline & (5) & 8.596 & 23.039 & 0.857 & 0.748 & $-14.31 \%$ & $-25.17 \%$ \\
\hline \multirow{5}{*}{$\mathrm{Ks}_{12}$} & (1) & 1.712 & 1.445 & 1.085 & 1.075 & $8.50 \%$ & $7.50 \%$ \\
\hline & (2) & 1.295 & 1.073 & 1.056 & 1.047 & $5.60 \%$ & $4.70 \%$ \\
\hline & (3) & 1.165 & 1.051 & 1.000 & 1.000 & $0.00 \%$ & $-0.01 \%$ \\
\hline & (4) & 2.451 & 2.465 & 0.923 & 0.918 & $-7.69 \%$ & $-8.21 \%$ \\
\hline & (5) & 8.747 & 8.835 & 0.786 & 0.784 & $-21.38 \%$ & $-21.61 \%$ \\
\hline \multirow{5}{*}{$\alpha 1$} & (1) & 1.684 & 1.684 & 0.999 & 0.999 & $-0.10 \%$ & $-0.09 \%$ \\
\hline & (2) & 1.376 & 1.376 & 0.999 & 0.999 & $-0.11 \%$ & $-0.10 \%$ \\
\hline & (3) & 1.202 & 1.202 & 0.999 & 0.999 & $-0.10 \%$ & $-0.10 \%$ \\
\hline & (4) & 0.914 & 0.914 & 0.999 & 0.999 & $-0.09 \%$ & $-0.09 \%$ \\
\hline & (5) & 0.834 & 0.834 & 0.999 & 0.999 & $-0.09 \%$ & $-0.09 \%$ \\
\hline \multirow{5}{*}{$\alpha 2$} & (1) & 2.061 & 2.062 & 0.992 & 0.993 & $-0.76 \%$ & $-0.70 \%$ \\
\hline & (2) & 1.527 & 1.528 & 0.997 & 0.997 & $-0.29 \%$ & $-0.30 \%$ \\
\hline & (3) & 1.166 & 1.168 & 0.999 & 1.000 & $-0.09 \%$ & $0.00 \%$ \\
\hline & (4) & 0.949 & 0.949 & 1.000 & 1.000 & $-0.01 \%$ & $-0.02 \%$ \\
\hline & (5) & 0.677 & 0.677 & 1.001 & 1.001 & $0.10 \%$ & $0.10 \%$ \\
\hline \multirow{5}{*}{$\mu_{11 \max }$} & (1) & 0.889 & 0.867 & 1.020 & 1.018 & $2.00 \%$ & $1.80 \%$ \\
\hline & (2) & 1.050 & 1.033 & 1.014 & 1.011 & $1.40 \%$ & $1.10 \%$ \\
\hline & (3) & 1.263 & 1.265 & 0.999 & 0.995 & $-0.14 \%$ & $-0.49 \%$ \\
\hline & (4) & 1.424 & 1.435 & 0.990 & 0.986 & $-1.04 \%$ & $-1.38 \%$ \\
\hline & (5) & 1.672 & 1.691 & 0.979 & 0.976 & $-2.08 \%$ & $-2.40 \%$ \\
\hline \multirow{5}{*}{$\begin{array}{c}\text { All } \\
\text { Param. } \\
\text { VFA } \\
\text { High }\end{array}$} & (1) & 3.716 & 24.697 & 1.126 & 1.391 & $12.60 \%$ & $39.10 \%$ \\
\hline & (2) & 2.626 & 2.252 & 1.080 & 1.086 & $8.00 \%$ & $8.60 \%$ \\
\hline & (3) & 2.181 & 2.654 & 0.935 & 0.921 & $-6.49 \%$ & $-7.91 \%$ \\
\hline & (4) & 12.669 & 13.237 & 0.724 & 0.728 & $-27.63 \%$ & $-27.21 \%$ \\
\hline & (5) & 20.360 & 34.183 & 0.652 & 0.556 & $-34.85 \%$ & $-44.43 \%$ \\
\hline \multirow{5}{*}{$\begin{array}{c}\text { All } \\
\text { Param. } \\
\text { VFA } \\
\text { Low }\end{array}$} & (1) & 3.716 & 24.697 & 1.126 & 1.391 & $12.60 \%$ & $39.10 \%$ \\
\hline & (2) & 2.626 & 2.252 & 1.080 & 1.086 & $8.00 \%$ & $8.60 \%$ \\
\hline & (3) & 2.181 & 2.654 & 0.935 & 0.921 & $-6.49 \%$ & $-7.91 \%$ \\
\hline & (4) & 12.669 & 13.237 & 0.724 & 0.728 & $-27.60 \%$ & $-27.21 \%$ \\
\hline & (5) & 20.360 & 34.183 & 0.652 & 0.556 & $-34.85 \%$ & $-44.43 \%$ \\
\hline
\end{tabular}

Table S2. Closed-loop performance of the standard and robust NMPC designs against

parameter uncertainties. 